plug. Two days later the gauze plug was removed without any further bleeding. The patient did not vomit after the second anæsthetic.

The patient was evacuated to the base on the tenth day, and a month after the injury I heard that he was doing well in England.

I have heard of other cases of wounds involving the inferior vena cava being brought to clearing stations, but I believe they have all ended fatally. The second of my two cases shows that it may be possible to save some of these patients by using forcipressure, or possibly suture in cases where the bleeding can be controlled while the suture is inserted. The reason that these cases do not bleed to death immediately is that the wounds in the posterior peritoneum and the cava are not, or do not remain, directly superimposed.

I am much indebted to Lieutenant-Colonel J. W. Longstaff, R.A.M.C., for permission to publish these notes, and also to Colonel H. M. Rigby, A.M.S., for his advice in the after-treatment of the second case.

\section{ON THE USE OF TUBERCULIN IN GENERAL PRACTICE.}

BY J. LINTON BOGLE, M.D. EDIN.,

LATE TUBERCULOSIS OFFICER UNDER THE COUNTY COUNCIL (PARTS OF LINDSEY), LINCOLNSHIRE.

GraNiEd the two postulates (1) that tuberculin is a useful remedy in the treatment of tuberculosis, and (2) that the use of tuberculin should not be the exclusive rôle of the specialist, but should belong to the armamentarium of the general practitioner, then notes on the main principles of tuberculin administration and hints as to the best practical means of carrying them out should be useful in inducing many medical men in general practice to commence treatment with this valuable remedy in the prevention and cure of the most generally prevalent disease in our country.

The best period to commence a course of treatment is in the early stages of the disease, at a time when only the family medical attendant is aware of its existence, and when a wise use of the proper remedies means all the difference between a vigorous and useful manhood on the one hand and a wasting and lingering malady on the other, followed too often by an early death. My experience as a tuberculosis officer has led me to conclude that amongst medical men generally there is a reluctance to venture on the use of tuberculin, based partly on the knowledge that, carelessly given, it is a potent cause of evil, and partly also on ignorance as to the proper ways and means to begin and to carry out the course of treatment. The notes are designedly simple; they are written for the instruction of those who have not hitherto used this method of treatment.

General Prinoiples of Tubereulin Administration.

Tuberculin, if injected into the healthy, produces no symptoms whatever, but in a tuberculous subject it causes the symptoms of the tuberculin reaction. The theoretical explanation of this reaction is that the tuberculous have present in their tissues a specific antibody or lysin, capable of breaking down the tuberculin molecule with the formation of toxins. After injection these toxins are carried into the general circulation, giving rise to the symptoms of general or focal reaction, and are followed by increase in the blood of antituberculous lysin or antibody, causing a state of tolerance or increased resistance to the effects of the tuberculous poison.

In the administration of tuberculin one of two courses is followed: either (1) it is given in small doses repeated at long intervals, cansing immunisation ; or (2) in initial small doses, advancing rapidly to large and repeated ones, producing immunisation with tolerance. The former is the course taken in glandular tuberculous disease in children, and local tuberculosis; and the latter in autotoxic disease, or pulmonary tuberculosis. When Koch first discovered the tubercle bacillus, and introduced his preparation of "Old Tuberculin," I remember administering the remedy under the advice of a Liverpool consultant. The usual mode of treatment at that time was large and increasing doses, causing severe reactions. As a result, the remedy quickly fell into disrepute. Now the aim of the medical man is the avoidance of reactions, or their production in a very mild degree, as a valuable guide to further treatment.

\section{Varieties of Tubercutin.}

All preparations of tuberculin, whether derived from the human or the bovine bacillus, have similar curative properties. They vary, however, in their strength and character. The preparations I have generally used are P.T.O., or bovine tuberculin; T.A.F., or tuberculin albumose-free ; and T., Koch's Old Tuberculin, belonging to the class of soluble extractives. Also B.E., bacillus emulsion, and P.B.E., bovine bacillus emulsion, belonging to the class of mixed products-i.e., soluble extractions, with the insoluble parts of the tubercle bacillus in addition. Their relative strength is approximately as follows, taking $\mathrm{T}$. as a standard: T. $=1$, T.A.F. $=1$, B.E $=\frac{1}{2}$, P.B.E. $=\frac{1}{2}$, P.T.O. $=1 / 10$.

\section{Preparation of Dilutions.}

The Record tuberculin syringe consists of a glass cylinder with a capacity of 1 c.c., or 16.9 minims, and is divided into tenths-i.e., divisions of 0.1 c.c. The syringe is filled with tuberculin to the first division, the remaining nine divisions are filled with diluent consisting of normal saline with 0.25 per cent. of carbolic acid. This is the first dilution, or Dil. A, and consists of 0.1 c.c. of tuberculin in 1 c.c. of dilution, or 1 in 10. Dil. B, or the second dilution, consists of one division or $1 / 10$ of Dil. A, together with $9 / 10$ of diluent ; it has 0.01 c.c. of tuberculin in 1 c.c., or 1 in 100 . Dil. $C$ in like manner has 0.1 c.c. of Dil. B to 0.9 c.c. of diluent, and $=0.001$ of tuberculin in 1 c.c., or 1 in 1000 . Dil. D has 0.0001 c.c. of taberculin in 1 c.c., and Dil. $\mathbb{E}$ has 0.00001 c.c. in 1 c.c., being respectively 1 in 10,000 and 1 in 100,000 of tuberculin in 1 c.c. of dilution. It is convenient to make these dilutions in bottles containing 10 c.c. (Sir A. E. Wright's vaccine bottles), and these bottles have rubber caps through which the needle can be plunged into the dilution without exposing it to the air.

\section{Mode of Administration.}

The usual method is by subcutaneous injection, although it is also given orally, in which case the dose is usually double that given under the skin; but as there is some doubt whether the gastric juices do not change and weaken the action of the tuberculin, the former method is the one in general use. It is to be preferred for its quick action, its accurate dosage, and also for the guidance given by the local reaction. In non-pulmonary tuberculosis the injection is sometimes made near the site of the diseased gland or joint. In oral administration the preparations used are T.R. or B.E.; they are given in a wineglassful of cold water an hour before a meal.

\section{The Tuberoulin Most Suitable for Children.}

In my experience P.B.E. (the bovine bacillus emulsion), B.E., and, on account of its relative mildness, P.T.O. are the most useful preparations. They give a slow and mild reaction, and it is here that Wright's system of repeated small doses spread over a long period is so valuable. In adults, where pulmonary disease is the usual type, it is advisable to begin with T.A.F. or P.T.O. and to follow, as a second course, with B.E. The first few doses are carefully watched, but usually afterwards they can be rapidly increased until the optimum dose is reached. Some very useful guidance can be obtained by observing carefully the symptoms of the tuberculin reaction. A local reaction, shown by a thickening or swelling in the tissues, with some tenderness, or by redness of the skin, is a warning that the dose is being increased quickly enough, and may soon produce a general reaction. A strong general reaction, shown by raised temperature, sickness, shivering, headache, and malaise, and, focally, by signs of crepitation or hæmorrhage in a diseased lung, points clearly to the dose being too high, and it should be reduced at once.

The main guidance in treatment by tuberculin is to be obtained from a regular observance of the temperature (the opsonic index being left to experts). It should be taken twice daily, at 830 A.M. and 6 P.M., and recorded upon a chart. In all rises of temperature rest is the primary necessity; a week in bed is most beneficial, especially in the early febrile stages of the disease.

\section{Dosage.}

Dosage of tuberculin varies according to the type of the disease. In local tuberculosis in adults an initial dose of 0.00005 , 1/20000 c.c., of P.T.O. or B.E., and in children 
one of $0.00002,1 / 50000$ c.c., of the same preparations, are fair average commencing doses. In pulmonary phthisis a dose of 0.000002 c.c. to 0.000005 c.c. of P.T.O., B E., or P.B.E. is sufficient. The sequence of doses is more rapid at first than afterwards. At first every four days, then weekly. After the first few doses the quantity is doubled.

The two following sequences, actually carried out in cases of tuberculous disease in children, are instructive :

1. Boy, aged 10, whose grandfather and two brothers had died from pulmonary tuberculosis, was found to be suffering from tuberculous disease of the glands and bones, having one sinus in the right popliteal space, two sinuses above the right ankle, four openings in the right groin (two discharging freely), two also in the left groin ; glands swollen and suppurating on left side of neck. On June 6th, 1914, the initial dose was 0.000001 c.c. P.B.E., and in February, 1915, he was receiving Dil. $D, 0.00001$ c.c. P.B.E., followed at intervals of two to three weeks by Dil. D, 0.2, $0.4,0.6$, Dil. C, $0.1,0.2,0.3,0.4,0.45,0.5,05,0.6,0.6,0.6,0.6$. His present condition is very satisfactory; all the sinuses are soundly healed, the only remnants of extensive disease from which he suffered being some fibrous scars in the groins and two small fibrous knots on the left and one palpable gland on the right side of the neck. His general health is excellent, with an increase in weight from 4 st. $1 \mathrm{lb}$. to 5 st. 1 lb.

2. Girl, aged 12. Commencing pulmonary tuberculosis. Had lived in the same house as her uncle, who died of the same disease a few months previously. She reacted to the von Pirquet test, and showed signs of commencing disease of the right lung. During the first few injections focal reaction occurred, shown by crepitant râles, altered breath sounds, and fever. Now she has no signs, having lost her cough and other symptoms, and is also gaining in weight. Initial dose, P.T.O. 0.000002 , Dil. $\mathrm{E}, 0.2,0.4,0.8 ; \mathrm{D}, 0.1,0.2$, $0.3,0.5$ (focal reaction), $0.35,0.25,0.35,0.70 ; \mathrm{C}, 0.1,0.2,0.3$, $0.4,0.5,0.65,0.75$.

Final dose.-The final dose of 0.1 c.c., or the whole c.c of Dil. A, is generally considered the highest safe dose-i.e., $1 / 10$ c.c. of tuberculin and $9 / 10$ diluent, but many medical men do not advance so far, being content with $0.5 \mathrm{~A}$, or $0.75 \mathrm{~A}$. In these higher doses, where the tendency to reaction is more manifest, it is also desirable to lengthen the interval, making it 10 to 14 days in place of a week; and after a few doses either to discontinue treatment for a few months or to inject a dilution of a different preparation of tuberculin of a lower strength. The initial dose of this new course may be of a higher strength than that of the first course on account of the state of tolerance established in the patient.

\section{Errors to be Avoided.}

It is necessary to remember that in passing from one dilution to another the strength is 10 times greater. Thus a sequence $0.5 \mathrm{D}$ to $0.75 \mathrm{D}$ is an increase of 50 per cent., but from $\mathrm{C} 0.1$ to $\mathrm{C} 0.2$ is 100 per cent. increase; so that on changing to a smaller injection of greater strength the increases should be small. Again, dilutions newly made are stronger than old dilutions, and the extractive toxin tuberculin dilutions are less stable than those made from the ground bacillary bodies, such as T.R. It is desirable to make fresh dilutions every two or three weeks in the former case, and every four or six weeks in the latter, or they will lose in strength.

The duration of the course depends upon the patient and the nature of the disease. A nervous patient, tending to high temperatures or hæmorrhage, requires smaller doses, and of a slowly-acting preparation, such as B E., and hence the course will be lengthened. In children, too, with glandular disease, and in all cases of bone disease. the course will be a long one, and much patience is required. In pulmonary disease of the ordinary early type the course would last from three to six months, with doses at intervals of 'a week. Then, after three months' rest, a shorter course of three months with another preparation of tuberculin. While undergoing this special treatment many patients can and do resume their occupations. The two following examples with their sequences of doses are interesting :-

(a) Young man aged 21. Pulmonary tuberculosis. Is now at work in a shed (outside) all day at his trade, the local signs having passed away. Once during my absence a dose many times stronger was given by inadvertence, with a resulting general reaction and a temperature reaching 104.2 F. Sequence: Dil. D, P.T.O., 0.5, 0.5; C, 0.1, 0.2, 0.4, $0.55,0.75 ; \mathrm{B}, 0.1,0.25,0.5,0.5,0.75$ (marked reaction); $\mathrm{B}, 0.1$ $0.2,0.3,0.4,0.5,0.5,0.4,0.5,0.55,0.75 ; \mathrm{A}, 0.1,0.25,0.3,0.4$,
$0.4,0.5,0.5,0.55,0.6,0.7,0.75,0.75,0.8$; reaction to $99.8^{\circ} \mathrm{F}$ Change to P.B.E. Dil. C, $0.1,0.15,0.25,0.35,0.5,0.75 ; \mathrm{B}, 0.1$, $0.15,0.2,0.3,0.35,0.35 ;$ P.B.E., 0.35 c.c. (here the error occurred). Dil. B, $0.35,0.5,0.75 ; \mathrm{A}, 0.1,0.15$; reaction to $99 \cdot 2^{\circ} \mathrm{F}$. once. This second course is now closed.

(b) Young man aged 27, shop assistant. Cavity in right apex, with consolidation of upper lobe of right lung and hæmoptysis. Had been in sanatorium for three months. The commencing dose was Dil. D, 0.00001 c.c. of P.B.E. $\mathrm{D}, 0.1,0.2,0.4,0.7 ; \mathrm{C}, 0.1,0.2,0.35,0.5,0.75 ; \mathrm{B}, 0.1,0.2,0.3$, $0.45,0.7 ; \mathrm{A}, 0.1$, slight hæmoptysis, rest from business for two weeks; one weekly injection omitted, then followed by A, $0.15,0.15,0.25,0.2$. He is working daily at his business and has had no higher temperature than $98^{\circ} 6^{\circ} \mathrm{F}$. since the first injection. Pulmonary symptoms quiescent and disease gradually drying up.

Tests for Tuberculosis.

Von Pirquet's test (cutaneous) is the most readily applied, the most satisfactory in its results, and the one generally used. This test is carried out as follows. The points of the scarifier having been sterilised by heating red-hot in the flame of the spirit lamp, a few scratches are made, as a control, upon the outer extensor surface of the arm or forearm, which has previously been carefully cleansed, and at a spot a few inches above, a drop of T. (Old Tuberculin) is, with the syringe, dropped upon the skin. The scarifier makes a few cross scratches under the tuberculin until a faint redness is perceptible. Tuberculin can be used pure or diluted, the rule being that the lower the strength used, to cause a reaction, the more active the disease. In 36 or 48 hours afterwards a note is taken of the temperature and of the condition of the punctured spots. If the result be positive the temperature may rise and the tuberculin spot become red and inflamed, with perhaps even a vesicular areola.

Contra-indioations to the Use of Tuberoulin.

Although experts, with their knowledge and wide experience, may be able to use tuberculin in most cases of tuberculosis with benefit and without injury, there are cases in which the general practitioner would do well to avoid the use of this remedy. In mixed infections, catarrhal or bronchial, or complicated by disease of the heart or kidneys ; in rapidly advancing or extensive disease ; in cases of high fever and quick pulse ; in anæmia associated with little power of resistance; in those in whom there is a strong hæmorrhagic tendency; and in cases of infantile tuberculosis, tuberculin as a rule does no good.

Although I have confined my remarks almost exclusively to tuberculin administration in treatment, it must never be lost sight of that unless a patient understands the advantage of life in the open air with abundance of light and sunshine, rest, healthy surroundings, the avoidance of excitement, and the value of good and nourishing food, with cod-liver oil and other food tonics, tuberculin alone can do very little. It is only in conjunction with a rational general treatment that the striking results of the special treatment are manifest. It is here that residence in a sanatorium does so much: it teaches the tuberculous patient how to live.

"Festina lente" is the golden rule in tuberculin administration, as experiments have shown that even such a minute dose as $1 / 1,000,000$ c.c. of tuberculin can raise the opsonic index from 0.70 to $1 \cdot 3$, although not the slightest clinical symptoms of change were present.

A word, in conclusion, as to the cost of the apparatus required. A good tuberculin syringe with two steel needles can be obtained for $9 s .6 d$. Tuberculin in its different preparations varies in price from $1 s$. to $3 s$. a bottle, containing 1 c.c. The scarifier costs only a few shillings, and the adjuncts are easily obtained at any chemist's. Altogether a very simple outlay.

Alexandra Rose Day was observed at Portsmouth on June 24th, when $£ 928$-a record sum-were collected on behalf of the Royal Hospital and the Eye and Ear Hospital.

CLERICAL, MEDICAL, AND GENERAL LIFE Assurance SocIETY.-The wax has certainly had no deteriorating effect on the business of this well-known society. The new net life business for the financial year ended June 30th was $£ 737,472$, being an increase of $£ 64,740$ over that of last year, with new premiums of $£ 25,792$, showing an increase of £1953. The amount of the claims was $£ 300,341$, being a decrease of $£ 48,710$ as compared with the previous year. Included in this amount is $£ う 4,148$ paid for war claims. 


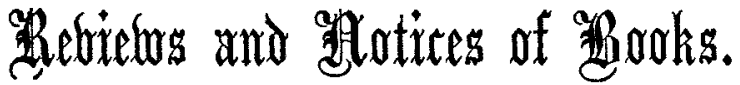

\author{
Burdett's Hospitals and Charities, 1916.
}

By Sir Henry Burdets, K.C.B., K.C.V.O. London : The Scientific Press, Limited. Price 10s. 6d. net.

THLs is an authoritative guide to British, American, and Colonial hospitals and asylums, medical schools and colleges, nursing and convalescent institutions, consumption sanatoria, and other institutions of a similar character, giving a review of their position and requirements, management, revenue, and cost of maintenance, and there is no reference book more convenient and useful than "Burdett's Hospitals and Charities." The present edition is on the lines of its predecessors, among the additional matter being a list of London institutions affording facilities for post-graduate work and a list of territorial hospitals. The great hospital funds of London, the institutions for the care of the blind, deaf, and dumb, orphanages, missions, nursing and convalescent institutions are all described, while there are chapters on hospital finance and hospital charity generally. The magnitude of the interests touched upon can be realised by the statement that in the year 1914 the 1978 charities of which particulars are forthcoming had an income from all sources of $£ 12,907,000$, or a decrease of $£ 651,000$ from the income for 1913. The expenditure in 1914 was $£ 12,144,000$ as compared with $£ 12,280,753$ in 1913. The expenditure on the management of hospitals will, of course, be enormously increased as a consequence of the war, while accommodation for the wounded has multiplied throughout the country, representing 100,000 additional beds. Sir Henry Burdett pays a well-deserved tribute to the way in which the voluntary hospitals have responded to the demands made upon them in spite of the heary financial drain on institutional resources. It is interesting to note that the Norfolk and Norwich Hospital has obtained a new source of revenue by an appeal in the form of 200 written letters, costing one sovereign, for contributions of $£ 5$ per month for each of the soldiers' beds. The response produced £5721. The beds are maintained by individuals and groups of persons of all classes, domestic servants contributing no less than £152. It is hoped that this systern will produce a steady revenue after the war.

In his preface Sir Henry Burdett refers to the fact that 50 years of continuous work for the voluntary hospitals of this country will be completed should he live until August, 1918, by which time he will have passed the allotted span of threescore years and ten. For several years in succession he has appealed for someone to take up and continue his task as editor, and it is to be regretted that there has been no response to the appeal. We hope that Sir Henry Burdett will be spared to edit for a long time yet a book of the utmost value to everyone interested in hospital charities, but it would be a great loss to the hospital world if from any cause the book which has been regularly issued for 27 years should fail to make its appearance.

\section{Nowveau Traité de Chirurgie.}

Publié sous la direction de A. Le Dentu et Pierre DelBet. Fractures. Vol. I. : Fractures en Général; Fractures du Membre supérieur. Par JEAN Tanton, Médecin-major de l'Armée, Professeur agrégé du Val-deGrâce. Avec 470 figures dans le texte. Paris: J. B. Baillière et Fils. 1915. Pp. 860 . Price 20 francs.

THIS fasciculus of an important treatise on surgery has appeared in spite of the war, and it is of special value when in numerable fractures present themselves to all those surgeons who are concerned with the treatment of the wounded. A glance at the volume is sufficient to show the thorough way in which the subject of fractures has been treated. Fractures of the skull, of the vertebral column, and of the thorax have been excluded, as they have been treated in other fasciculi of this system of surgery, while the author has been unable to include in this volume more than the general subject of fracture and the fractures of the upper limb. The fractures of the lower limb and of the pelvis will form the subject of another volume. The recent great increase in our knowledge of fractures has been undoubtedly due to the introduction of the $X$ rays, for thus we have been enabled to gain exact knowledge of the pathology of the living; and the author has appreciated to the full the value of skiagrams, for he has included in his work a large number. $\mathrm{He}$ is, however, fully alive to the erroneous deductions which the untrained may draw from $X$ ray photographs, and he shows us illustrations exemplifying this fact.

It is needless to criticise in detail such a work as this when we are able to draw attention to the completeness and exactitude with which the author has dealt with the whole subject. We cannot give a better example of his thoroughness than his treatment of fracture of the base of the first metacarpal bone, for he recognises three, or rather four, forms of this fracture, and each of these varieties is illustrated with photographs. The sections dealing with the treatment of the various fractures are good; the illustrations are abundant and apposite.

We have not met a more scholarly treatise on fractures, and we look forward with interest to the appearance of the next volume.

The Origin and Nature of the Emotions: Miscetlaneous Papers.

By George W. Crile, M. D., Professor of Surgery, School of Medicine, Western Reserve University, Cleveland. Edited by AMX F. Rowland, B.S. London and Philadelphia: W. B. Saunders Company. 1915. Pp. 240. Price 13s, net.

Dr. Crile's work on shock, anoci-association, and kindred topics is already well known in this country. The present volume contains various papers that bear more or less on his previous contributions ; they serve also as a preface to certain monographs which we are told are in preparation. It is not practicable to give an analysis of all the eight essays comprising this volume; they deal with such diverse subjects as the relation between the physical state of the brain-cells and brain functions, alkalescence, acidity and anæsthesia, pain, laughter and crying, a mechanistic view of psychology, a mechanistic theory of disease-to name but some of them. We may refer more particularly to those which have a bearing on the title of the book.

Briefly, it may be stated that the author has determined by laboratory researches that such emotions as fear and anger produce the same histological changes in the brain as physical trauma. Inasmuch as the phylogenetic origin of fear is injury, it follows that injury and fear cause the same phenomena. In their quality and in their expression, psychic shock and physical shock are the same. Dr. Crile has made a patient investigation of the cells of the cortex in human cases of exhausting diseases, in various animals completely exhausted by running and fighting, by rage and fear, by physical injury, and by the injection of strychnine, and in every instance he has been able to demonstrate that the loss of vitality-in other words, the loss of the normal power to convert potential into kinetic energy-is accompanied by physical changes in the brain cells. He concludes that these changes are "work" changes. But, further, changes were also found in the adrenals and in the liver, again in every instance. Dr. Crile regards these three organs as constituting what he calls the "kinetic system." Linked to it is the thyroid gland. "When the kinetic system is driven at an overwbelming rate of speed, as by severe physical injury, by intense emotional excitation, by perforation of the intestines, ...... by the sudden onset of an infectious disease, by an overdose of strychnine, by a Marathon race, by a grilling fight, ..... by anaphylaxis, the result of these acute overwhelming activations of the kinetic system is clinically designated shock, and according to the cause is called tranmatic shock, toxic shock, anaphylactic shock, drug shock, \&c." In this way the author endeavours to harmonise the pathological changes in these organs in disease with the occurrence of the same changes as the result of emotional strain. Conversely, any agency that can sufficiently inspire faith or dispel worry will inhibit the action of emotion on these organs. They can be, and are, physically damaged by fear-they can be protected and strengthened in their function by suitable psychical stimuli.

We commend to the thoughtful reader these essays on the fascinating subject of psychical and physical interaction. 